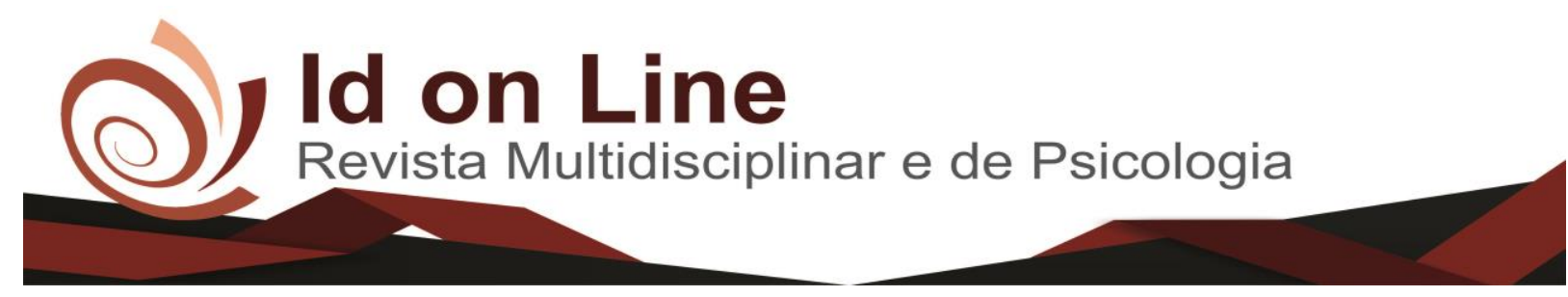

Artigo

\title{
Efeitos da Mobilização Neural na Incapacidade Funcional em Pacientes com Hérnia de Disco Cervical
}

\author{
Virgilio Santana Júnior ${ }^{\text {; }}$ Tácila Ribeiro Coelho ${ }^{2}$
}

Resumo: O objetivo deste estudo foi analisar o efeito da Mobilização Neural em pacientes com hérnia de disco cervical. Trata-se de um estudo descritivo de corte transversal com abordagem quantitativa, realizado com dois indivíduos, sendo um do sexo masculino e outro do sexo feminino. Aplicou-se um questionário sociodemográfico, questionário de McGill e a Escala Visual Analógica para a identificação das variáveis do estudo. O tratamento foi feito com a Mobilização Neural em dezesseis sessões. Pode-se observar um decréscimo da percepção de dor através da Escala Visual Analógica. Por meio da aplicação do McGill foi possível evidenciar que o subgrupo de maior índice de dor nos dois indivíduos foi o de experiência dolorosa. Na avaliação da goniometria evidenciou crescimento constante da média de angulação do indivíduos avaliados para todas as categorias de manobras. A mobilização neural no tratamento de pacientes com hérnia de disco cervical mostrou ser eficaz.

Palavras-chave: Hérnia de Disco Cervical. Incapacidade Funcional. Mobilização Neural.

\section{Effects of Neural Mobilization on Functional Impairment in Patients with Cervical Disc Herniation}

\begin{abstract}
This study aimed to analyze the effect of Neural Mobilization in patients with cervical disc herniation. This is a cross-sectional descriptive study with a quantitative approach, carried out in two individuals, one male and one female. A sociodemographic questionnaire, McGill Pain Questionnaire and the Visual Analog Scale were used to identify the study variables. The treatment was performed with Neural Mobilization in sixteen sessions. A decrease in the perception of pain through VAS can be observed. Through the application of the McGill Pain Questionnaire, it was possible to show that the subgroup with the highest pain index in the two individuals was the one with pain experience. In the evaluation of goniometry, there was a constant increase in the mean angulation of the individuals evaluated for all maneuver categories. Neural mobilization in the treatment in patients with cervical disc herniation has been shown to be effective.
\end{abstract}

Keywords: Cervical disc herniation. Functional disability. Neural mobilization.

\footnotetext{
${ }^{1}$ Fisioterapeuta. Docente da FAINOR e FTC em Vitória da Conquista/BA. Especialista em terapia manual e postural CESUMAR.

${ }^{2}$ Discente graduanda do curso de Fisioterapia pela Faculdade Independente do Nordeste - FAINOR. http://www.fainor.com.br, Vitoria da Conquista - BA, Brasil.
}

Autor correspondente: Virgilio Santana Junior, Rua Hormindo Barros, 244; Bairro Candeias; CEP 45029-094; Vitoria da Conquista/BA; virgiliofisio@ hotmail.com (77)98807-8862. 


\section{Introdução}

Dentre as diversas patologias que acometem a coluna vertebral, a hérnia de disco destaca-se por seu alto índice de incidência. Além de trazer transtornos psicossociais, pode gerar diversos impactos econômicos, tendo em vista que o indivíduo fica impossibilitado de trabalhar. A hérnia de disco é uma doença crônica degenerativa da coluna, gerada por uma lesão nos discos intervertebrais, sendo originada por diversos fatores (HELFENSTEIN et al., 2010).

A hérnia de disco cervical é uma protusão do núcleo pulposo do disco intervertebral podendo comprimir uma ou mais de uma raiz nervosa. A hérnia é menos comum de ser encontrada na cervical que na região lombar e é geralmente encontrada em C5-C6 ou então entre C6-C7, sendo C2-C3 mais raras, isto porque há uma sobrecarga nesses níveis. (GABRIEL; PETIT; CARRIL, 2001).

Comumente, o paciente com hérnia de disco cervical irá sentir dores gravíssimas, impedindo-os de ficar confortáveis em qualquer posição. Posteriormente, os sintomas vão piorando, como por exemplo: ao fazer uma extensão ou rotação da cabeça para o local onde está a dor. Espasmos musculares, fraqueza motora, alterações sensoriais em dermátomos específicos, redução da amplitude de movimento vertebral afetado, dor no pescoço, ombro, braço e na mão, também são sintomas encontrados na hérnia de disco cervical (DUTTON, 2010).

A movimentação ativa do pescoço fica afetada, principalmente em movimentos como extensão, rotação e inclinação lateral, independente se for para o a direção da raiz nervosa afetada ou não. Ao fazer uma inclinação lateral para o lado oposto afetado, isso irá aumentar a condução da hérnia de disco sobre a raiz nervosa, entretanto, essa inclinação também irá levar a uma dor, pois estará comprimindo a raiz nervosa no forame neural (DUTTON, 2010).

O tratamento para paciente com hérnia de disco deve ser feito de forma individualizada, respeitando a condição de cada um. Segundo Dutton (2010, p. 1552) "“[...] Muitos pacientes obtém analgesia com o uso controlado de retração cervical ou deslizamento posterior da coluna cervical inferior, em combinação com extensão da coluna cervical inferior e flexão da coluna cervical superior (queixo contraído) [...]”. Mas vale salientar que o uso desse exercício só deve permanecer em prática, até o momento em que o paciente esteja obtendo benefícios. Já a intervenção cirúrgica só é indicada quando o método conservador ultrapassa de 
8 semanas e não houve melhora alguma, aí o paciente é encaminhado para a cirurgia (DUTTON, 2010).

A técnica de mobilização neural tem como prioridade restaurar o movimento e a elasticidade do sistema nervoso, objetivando aperfeiçoar a neurodinâmica e reestabelecer o fluxo exoplasmático (PEREIRA; SCHONS, 2015). Estudos demonstram que esta técnica de mobilização neural age como tratamento de patologias que acometem o sistema nervoso. Outro estudo traz que a Mobilização Neural é um ótimo tratamento para cervicobraquialgia, pois aumenta a mobilidade articular da cervical e do ombro, lenvando ao final da (MONNERAT; NUNES, 2011).

A escolha por esse tema deveu-se à carência de estudos na área, além dessa técnica voltada para a região cervical ser pouco utilizada no cotidiano clínico dos fisioterapeutas.

O estudo tem por finalidade avaliar os efeitos da mobilização neural na incapacidade funcional de pacientes com hérnia de disco cervical.

\section{Metodologia}

A presente pesquisa foi do tipo descritivo de corte transversal com abordagem quantitativa, realizado em um Núcleo de Estudo de Fisioterapia da cidade de Vitória da Conquista - Bahia.

A amostra foi do tipo não probabilístico por conveniência composta por dois indivíduos do sexo masculino e feminino com idade de 72 e 48 anos respectivamente. Foram selecionados os pacientes que são atendidos no Núcleo de Estudo de Fisioterapia, que correspondiam aos seguintes critérios de inclusão: Exames de imagem que comprovam disfunções na coluna cervical, hérnia de disco cervical crônica e que tenham assinado o Termo de Consentimento Livre Esclarecido (TCLE). Já como critérios de exclusão foram selecionados: Pacientes que já foram submetidos à cirurgia na coluna cervical e/ou fraturas, hérnia de disco cervical na fase aguda e pacientes com distúrbios cognitivos que pudessem dificultar a compreensão dos instrumentos de pesquisa.

A pesquisa foi desenvolvida no período de setembro e outubro 2017, sendo dividido em dois atendimentos semanais. Como instrumentos para coleta dos dados foram utilizados: Ficha de Avaliação Ortopédica, Exame Clínico, Escala de Eva, Testes ortopédicos, Questionário de 
dor McGILL e Exames de imagem. Para o procedimento da Coleta de Dados, foram utilizados: Uma maca de $80 \mathrm{~cm}$ de altura, $70 \mathrm{~cm}$ de largura e $1.85 \mathrm{~m}$ de comprimento; e um goniômetro clínico da marca Fast Saúde.

A coleta dos dados foi realizada pelo avaliador e foi dividido em três períodos: Avaliação inicial, aplicação da mobilização neural e reavaliação. Durante a avaliação inicial, o avaliador preencheu a ficha de identificação de cada indivíduo e cada participante respondeu ao Questionário de dor McGILL e a Escala de EVA.

A Ficha de avaliação ortopédica foi criada pelo próprio pesquisador, nela irá conter: Data da avaliação, Anamnese, Avaliação clínico-funcional, Diagnóstico funcional, Objetivos, Conduta/Tratamento fisioterapêutico. A avaliação feita pelo fisioterapeuta tem como finalidade criar um banco de dados que permita identificar o grau de funcionalidade do paciente, identificar também o problema e os motivos que levaram o paciente a procurar o atendimento. Ainda, possibilita que sejam feitas comparações entre o primeiro e o último atendimento, com a finalidade de avaliar a eficácia do tratamento (AMADO, 2006, p.2).

A escala visual analógica (EVA) possibilita que seja feita uma medida simples e hábil da intensidade da dor. Ela vem sendo muito empregada em clínicas e hospitais. A EVA é instituída por uma linha de $10 \mathrm{~cm}$, que tem como inicio a pergunta sobre a ausência da dor e termina com dor insuportável. Essa escala pode apresentar um grau de dificuldade ao ser trabalhada com idosos e crianças, então, para uma melhor compreensão, as perguntas podem ser substituídas por desenhos de expressões faciais que caracterizam cada pergunta (MARTINEZ; GRASSI; MARQUES, 2011).

O questionário de dor McGill (MPQ), tem como finalidade avaliar as qualidades sensoriais, afetivas e temporais da dor. Esse questionário vem sendo muito bem aplicado na prática clínica. Apesar de ser uma ferramenta que oferece confiança nos seus resultados, ainda assim, se faz necessário ponderar a sua aplicação na população idosa, sendo que a dor é uma experiência multidimensional e pode sofrer influencia pelo processo de envelhecimento (SANTOS et al., 2006).

Em seguida, o avaliador posicionou o paciente em sedestação sobre uma maca para submetê-lo ao Teste de abdução de ombro que foi realizado da seguinte maneira: Paciente sentado, o avaliador eleva o braço em abdução de forma passiva ou pode ser feito de forma ativa, se ocorrer um alívio da dor, indica um problema de compressão extradural cervical, que pode ser um disco herniado ou compressão de raiz nervosa. Logo após a realização do teste o 
avaliador por meio do goniômetro mensurou o ângulo de flexão, extensão, flexão lateral direito e flexão lateral esquerdo da cervical.

Os participantes foram submetidos à mobilização neural para o nervo mediano que foi aplicado da seguinte maneira: Paciente em decúbito dorsal, o avaliador faz uma inclinação da cabeça para o lado contralateral do membro a ser realizado a técnica, realiza uma abdução de ombro em $90^{\circ}$, flexão de cotovelo, em seguida estabiliza o ombro faz uma rotação interna de ombro e uma extensão de cotovelo sem fazer oscilações. Foram realizadas três repetições durante um minuto, com intervalos de trinta segundos em repouso, a técnica foi aplicada bilateralmente, começando sempre pelo membro superior direito para uniformizar o estudo.

Logo após a aplicação da Mobilização Neural, foi realizada uma nova goniometria com o objetivo de verificar se houve alterações na amplitude de movimento pós Mobilização Neural do nervo mediano e os resultados eram anotados na ficha de identificação pessoal de cada paciente.

Para fins de geração dos resultados, os dados categóricos foram apresentados por meio de frequências relativas e absolutas, enquanto os contínuos através de médias e desvio padrões (quando necessário), dada a normalidade dos dados atestada pelo teste de Shapiro Wilk. A totalidade dos análises foi realizada no SPSS versão 21 e os gráficos plotados pelo Sigma Plot versão 12.0 .

O presente trabalho atendeu às normas para a realização de pesquisa em seres humanos e cumpriu as diretrizes da Resolução 466/12 do Conselho Nacional de Saúde, sendo o trabalho aprovado pelo Comitê de Ética da Faculdade Independente do Nordeste, n. 2132358.

\section{Resultados}

Entre os indivíduos avaliados $(\mathrm{n}=2)$ a média de idade foi de 60 anos $( \pm 17)$, tendo o homem duas hérnias de disco localizadas em C5/C6 e C6/C7 e a mulher apenas uma, situada em $\mathrm{C} 3 / \mathrm{C} 4$.

Os resultados da EVA mostraram uma tendência de decréscimo da percepção de dor tendo a média da avaliação pré manipulação reduzido de 8,25 na primeira semana para 2,25 na oitava. De forma similar a escala pós avaliação apresentou comportamento semelhante tendo 
decaído de 7,75 na primeira semana para 2,0 na oitava, conforme apresentada na figura 1 a seguir.

Figura 1. Escala analógica visual (EVA) para mensuração da dor pré e pós manipulação durante 8 semanas. Vitória da Conquista, 2017.

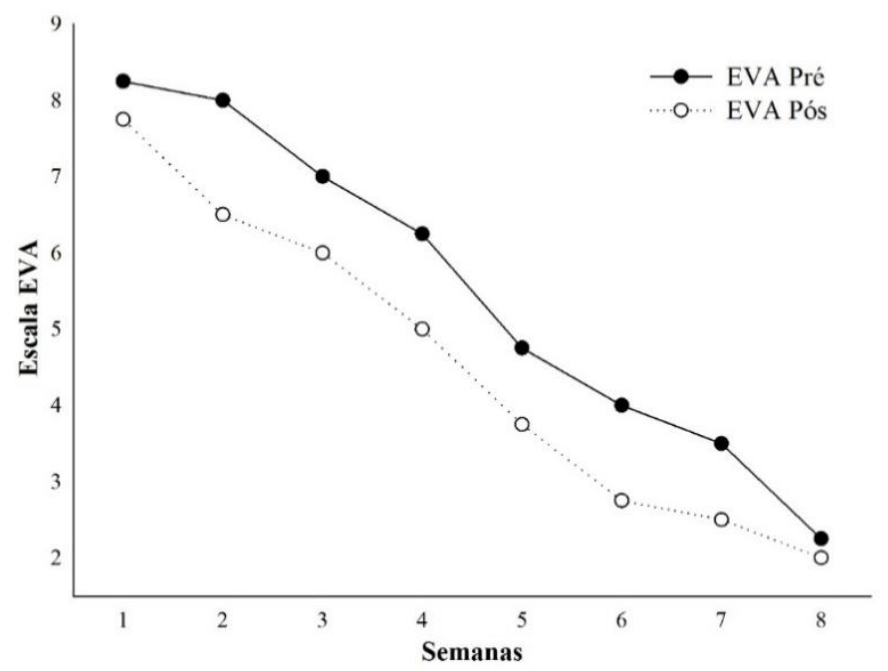

Fonte: Dados da pesquisa.

No questionário McGill, que expressa qualitativamente um descritor de sua experiência dolorosa, o maior número possível atribuído à intensidade da dor é 20. Este índice de dor é obtido pela soma dos valores de intensidade dos descritores escolhidos, e pode ser obtido para o total e para cada um dos quatro componentes do questionário: sensorial, afetivo, avaliativo e miscelânea (mista).

Por meio da aplicação do McGill foi possível evidenciar que o subgrupo de maior índice de dor nos dois indivíduos foi o de experiência dolorosa, ainda pela análise da tabela vislumbra-se que o indivíduo 1 obteve pontuação de 32 numa escala de máximo de 75 , enquanto o indivíduo 2, 36 pontos. O subgrupo de maior média foi o de "miscelânea" média de 3,7 ( $\pm 0,98)$, seguindo por "experiência dolorosa" $2,35( \pm 0,02)$, "avaliativo" $2,00( \pm 0,00)$; e "caráter afetivo" $1,33( \pm 0,47)$, os demais dados estão expostos na tabela 1 abaixo: 
Tabela 1. Descrição dos subgrupos do McGill de acordo com o índice numérico e de dor. Vitória da Conquista, 2017

\begin{tabular}{lcccc} 
& Índice numérico & Índice de dor & Média & dp \\
\hline Indivíduo 1 & 9 & 21 & 2,33 & 1,58 \\
Dolorosa & 4 & 4 & 1,00 & 0 \\
Afetivo & 1 & 2 & 2,00 & 0 \\
Avaliativo & 3 & 5 & 3,00 & 2,82 \\
Miscelânea & & & & \\
Indivíduo 2 & 8 & 19 & 2,37 & 1,50 \\
Dolorosa & 3 & 5 & 1,66 & 1,15 \\
Afetivo & 1 & 2 & 2,00 & 0 \\
Avaliativo & 4 & 10 & 4,40 & 4,50 \\
Miscelânea & & & & \\
\hline
\end{tabular}

Fonte: Dados da pesquisa

A avaliação da goniometria evidenciou crescimento constante da média de angulação do indivíduos avaliados para todas as categorias de manobras. A média pré flexão foi de $0,44^{\circ}$ na primeira semana; $0,46^{\circ}$ na segunda; $0,50^{\circ}$ na terceira; $0,53^{\circ}$ na quarta; $0,57^{\mathrm{a}}$ na quinta; $0,61^{\circ}$ na sexta; 0,64 na sétima; e 0,69 na oitava. Para as medidas pós manobra a variação foi de $0,45^{\circ}$ na primeira; $0,49^{\circ}$ na segunda; $0,52^{\circ}$ na terceira; $0,55^{\circ}$ na quarta; $0,59^{\circ}$ na quinta; $0,63^{\circ}$ na sexta; $0,68^{\circ}$ na sétima; e $0,73^{\circ}$ na oitava (Figura $2[\mathrm{~A}]$ ).

Figura 2 [A]. Média da goniometria pré e pós manipulação neural para flexão do pescoço ao longo de oito semanas

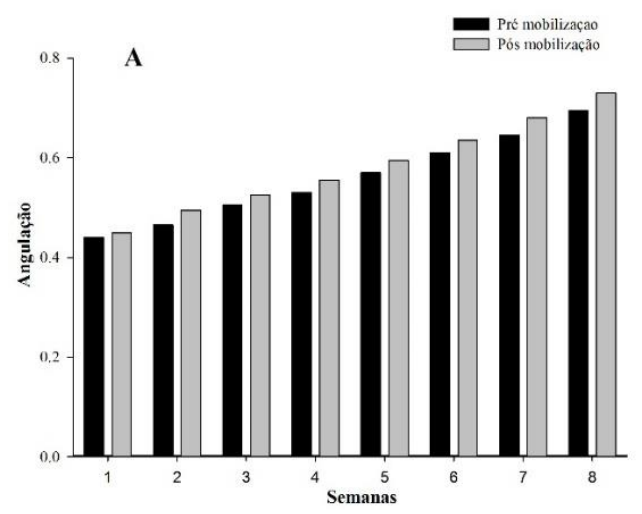

Fonte: Dados da pesquisa. 
Para a extensão a variação de média pré foi de $0,36^{\circ}$ na primeira semana; $0,37^{\circ}$ na segunda; $0,41^{\circ}$ na terceira; $0,45^{\circ}$ na quarta; $0,50^{\circ}$ na quinta; $0,56^{\circ}$ na sexta; $0,60^{\circ}$ na sétima; e $0,64^{\circ}$ na oitava. No pós manobra de a primeira semana apresentou média de $0,45^{\circ}$; a segunda $0,40^{\circ}$, a terceira $0,43^{\circ}$; a quarta $0,48^{\circ}$; a quinta $0,53^{\circ}$; a sexta $0,58^{\circ}$; a sétima $0,63^{\circ}$; e a oitava $0,66^{\circ}$ (Figura $\left.2[\mathrm{~B}]\right)$.

Figura 2 [B]. Média da goniometria pré e pós manipulação neural para extensão do pescoço ao longo de oito semanas.

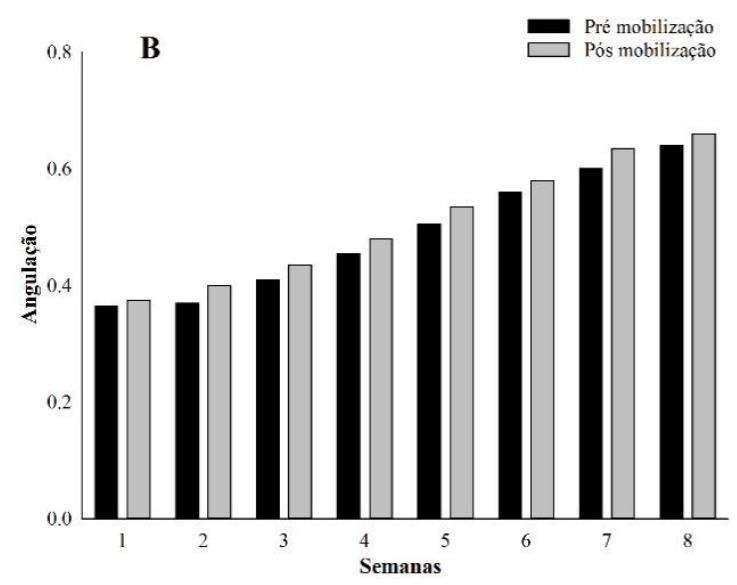

Fonte: Dados da pesquisa.

A média pré flexão lateral direita foi de $0,28^{\circ}$ na primeira semana; $0,29^{\circ}$ na segunda; $0,31^{\circ}$ na terceira; $0,34^{\circ}$ na quarta; $0,38^{\circ}$ na quinta; $0,41^{\circ}$ na sexta; $0,43^{\circ}$ na sétima; e 0,44 na oitava. Quando observada a média pós manobra, a mesma foi de $0,28^{\circ}$ na primeira semana; $0,31^{\circ}$ na segunda; 0,34 na terceira; $0,37^{\circ}$ na quarta; $0,40^{\circ}$ na quinta; $0,43^{\circ}$ na sexta; 0,45 na sétima; e $0,45^{\circ}$ na oitava (Figura $2[\mathrm{C}]$ ). 
Figura 2 [C]. Média da goniometria pré e pós manipulação neural para flexão lateral direita do pescoço ao longo de oito semanas.

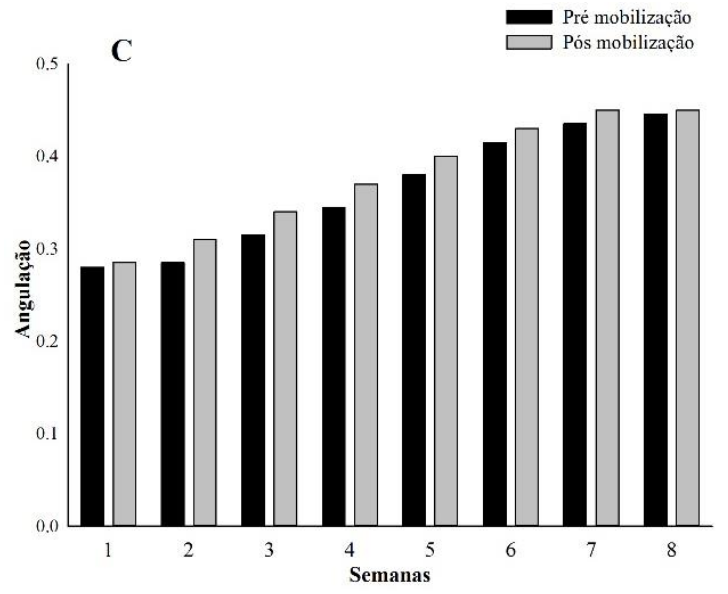

Fonte: Dados da pesquisa.

Por fim, a média pré extensão lateral esquerda na primeira semana foi de $0,26^{\circ} ; 0,28^{\circ}$ na segunda; $0,30^{\circ}$ na terceira; $0,33^{\circ}$ na quarta; $0,37^{\circ}$ na quinta; $0,41^{\circ}$ na sexta; $0,42^{\circ}$ na sétima; e $0,44^{\circ}$ na oitava. No pós manobra por sua vez, a média na primeira semana foi de $0,27^{\circ} ; 0,30^{\circ}$ na segunda; $0,33^{\circ}$ na terceira; $0,36^{\circ}$ na quarta; $0,39^{\circ}$ na quinta; $0,42^{\circ}$ na sexta; $0,44^{\circ}$ na sétima; e $0,44^{\circ}$ na oitava (Figura $2[D]$ )

Figura 2 [D]. Média da goniometria pré e pós manipulação neural para flexão lateral esquerda do pescoço ao longo de oito semanas.

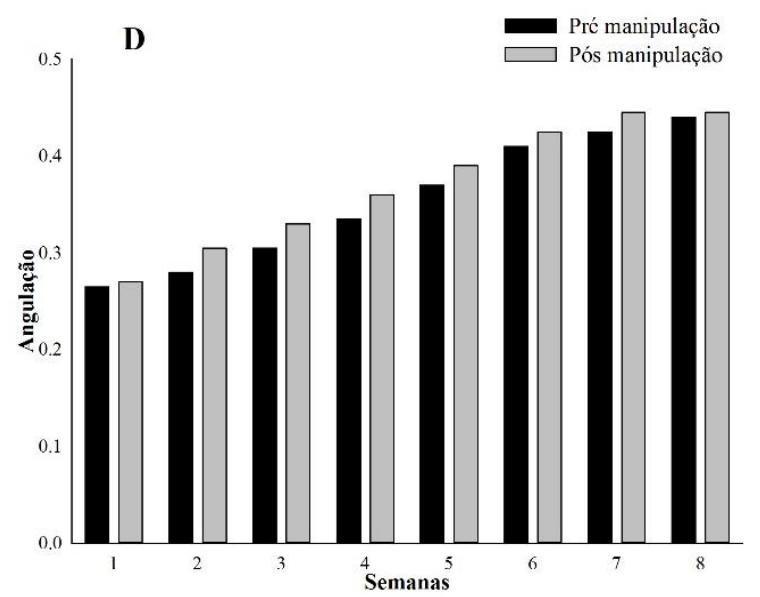

Fonte: Dados da pesquisa 


\section{Discussão}

A presente pesquisa objetivou investigar os efeitos da técnica de mobilização neural em pacientes com hérnia de disco cervical, avaliando a dor e amplitude de movimento pré e pós mobilização do nervo mediano. Não houve evidências estatísticas significativas, este fato pode estar relacionado ao número reduzido da amostra. Entretanto, é possível observar uma melhora na dor através dos valores obtidos pela EVA. Esse resultado corrobora com o estudo de (BARETA et al, 2017), que nos traz que a mobilização neural quando aplicada em pacientes com compressão nervosa, proporciona uma melhora na dor e uma melhora no quadro funcional.

Em um estudo realizado por Machado e Bigolin (2010) em dois grupos distintos com sujeitos que apresentavam lombalgia crônica, foram realizadas 20 sessões de um programa de mobilização neural e de um programa de alongamento muscular. Em relação ao quadro álgico através da avaliação da EVA, observou-se uma redução significativa nos indivíduos do grupo tratado com mobilização neural. Por sua vez, o alongamento muscular não diminuiu de forma significativa a dor do grupo que recebeu essa intervenção.

Ramiro (2016) em um estudo recente, concluiu que a dor e a perda de função de um paciente com hérnia de disco cervical, pode levar a um déficit funcional do mesmo. Além disso, as pessoas em geral estão com hábitos de vida tão desordenados que as hérnias de discos cervicais serão cada vez mais frequentes em nossa rotina. Mazzucco et al., 2017 analisaram a postura dos acadêmicos do curso de Odontologia durante os atendimentos. E comprovaram que há prevalência de dor na região cervical dos acadêmicos de odontologia devido a postura que eles adotam durante o atendimento.

No resultado do questionário McGill, verificou-se que o subgrupo de maior índice de dor nos dois indivíduos foi o de experiência dolorosa. Esse achado confirma o estudo de Pitangui et al, (2009) realizado em quarenta puérperas primíparas submetidas ao parto normal com episiotomia. Objetivou-se mensurar e caracterizar a percepção dolorosa. Os descritores que mais apresentaram a dor foram: dolorosa, que incomoda, chata, ardida, pica como uma agulhada, latejante e em pressão.

Embora a técnica de mobilização neural não age diretamente em músculos e fáscias, é notado um ganho de ADM após a sua aplicação (VASCONCELOS; LINS; DANTAS, 2011). 
No presente estudo foram verificados os efeitos da $\mathrm{MN}$ evidenciando um crescimento constante da média de angulação dos indivíduos avaliados para todos os movimentos mensurados através da avaliação goniométrica. Segundo Machado et al., (2015) o resultado do aumento da ADM pode estar ligado ao fato de o sistema nervoso ser visto como um trato tecidual único e a aplicação de tensão pode surtir efeitos de ampliação em qualquer parte desse sistema.

Barbosa e Leal (2015), em sua pesquisa avaliaram 50 indivíduos, divididos em dois grupos. No Grupo 1, foi realizada uma avaliação da ADM do membro inferior direito, em seguida, aplicaram a técnica de $\mathrm{MN}$ e reavaliaram a ADM também no referido membro. No Grupo 2, realizaram avaliação do membro inferior direito, aplicarão a técnica de $\mathrm{MN}$ no esquerdo e reavaliarão a ADM no lado direito. Foi observado que a média da ADM apresentou aumento significativo nos dois grupos, obtendo um maior resultado o grupo da mobilização indireta.

Como limitação deste estudo pode ser citado o fato do número de participantes ser restrito, além dos pacientes já portarem outras doenças de base na região cervical, influenciando na eficácia do tratamento. Vale ressaltar a importância de serem realizadas novas pesquisas relacionadas ao tema para que haja melhor aperfeiçoamento e validação desta técnica.

\section{Conclusão}

Neste estudo, a mobilização neural do nervo mediano demonstrou ser uma técnica eficaz para a redução do quadro álgico e o ganho da amplitude de movimento da cervical em indivíduos com hérnia de disco cervical, quando comparado o antes e o após a aplicação da técnica. Porém, não apresentou diferença estatisticamente significativa pelo tamanho reduzido da amostra.

\section{Referências}

AMADO, J.S.M. Métodos de avaliação clínica e funcional em fisioterapia. Rio de Janeiro: Guanabara Koogan, 2006. 
BARBOSA, A.P.B; LEAL, S.S. Análise da eficácia da mobilização neural do nervo isquiático sobre ganho de ADM. ConsSaud,.v.14, n.3, 2015.

CARVALHO, L.B. et al. Hérnia de disco lombar: tratamento. Acta Fisiatr, v.20, n.2, 2013.

DUTTON, M. Fisioterapia Ortopédica: Exame, Avaliação e Intervenção. Porto Alegre: Artmed, 2010.

GABRIEL, M. R. S; PETIT, J. D; CARRIL, M. L. Fisioterapia em traumatologia, ortopedia e reumatologia. Rio de Janeiro: Revinter, 2001.

MACHADO, A.F. et al. Efeitos imediatos e tardios da mobilização neural sobre força de preensão palmar e complacência neural de membro superior: um ensaio clínico randomizado. ConsSaude, v.14, n.3, 2015.

MACHADO, G.F; BIGOLIN, S.E. Estudo comparativo de casos entre a mobilização neural e um programa de alongamento muscular em lombálgicos crônicos. Fisioter. Mov, v. 23, n. 4, 2010.

MARTINEZ, J.E; GRASSI, L.G; MARQUES, D.C. Análise da aplicabilidade de três instrumentos de avaliação de dor em distintas unidades de atendimento: ambulatório, enfermaria e urgência. Rev. bras. Reumatol, v.51, n.4, p.304-308, 2011.

MAZZUCCO, A. et al. Posturas adotadas durante os procedimentos odontológicos e os seus impactos biomecânicos. Revista Inova Saúde, v.6, n.1, 2017.

MONNERAT, E; NUNES, P.C. Comparação dos tratamentos conservador, cirúrgico e através da mobilização neural no tratamento da hérnia de disco lombar. Fisioterapia Brasil, v. 13, n. $2,2012$.

PEREIRA, A.A; SCHONS, D.G. Os efeitos da Mobilização Neural em Pacientes com lombociatalgia. Rev Fisioter S Fun, v. 4, n. 2, p. 14-20, 2015.

RAMIRO, R. Hérnia de Disco Cervical Causas e Fisiopatologia. Revista Uniplac, v.4, n.2, 2016.

RODART, A.C. et al. Mensuração e característica da dor perineal em primíparas submetidas à epsiotomia. Acta Paul Enferm, v.22, n.1, 2008.

SANTOS, C.C et,al. Aplicação da versão brasileira do questionário de dor Mcgill em idosos com dor crônica. Acta Fisiatr, v.13, n.2, 2006. 
VASCONCELOS, D. A.; LINS, L. C. R. F.; DANTAS, E. H. M. Avaliação da mobilização neural sobre o ganho de amplitude de movimento. Fisioterapia e Movimento, v. 24, n. 4, p. 665672, out./dez. 2011.

\section{Como citar este artigo (Formato ABNT):}

SANTANA JÚNIOR, Virgílio; COELHO, Tácila R. Efeitos da Mobilização Neural na Incapacidade Funcional em Pacientes com Hérnia de Disco Cervical. Id on Line Revista ultidisciplinar e de Psicologia, 2017, vol.12, n.39, p.58-70. ISSN: 1981-1179.

Recebido: 12.11 .2017

Aceito: 16.11 .2017 